\title{
Interplay between TRa1 and Wnt signaling: A dangerous liaison
}

\section{Sheue-Yann Cheng}

News on: Increased expression of the thyroid hormone nuclear receptor TRa 1 characterizes intestinal tumors with high Wnt activity by Uchuya-Castillo et al. Oncotarget. 2018; 9:30979-30996. https://doi.org/10.18632/oncotarget.25741

In the past decades, great strides forward have been made in the understanding of genomic actions of thyroid hormone nuclear receptors (TRs). One aspect of TR actions that has received relatively little attention until recently is the role of TRs in carcinogenesis. There are two major thyroid hormone (T3) binding TRs-TR 1 and TR $\beta 1$. They share high sequence homology in the DNA and T3 binding domains, but they totally differ in the amino terminal A/B domain in the length and amino acid sequences. Increasing evidence from several groups of investigators has clearly demonstrated that TR $\beta 1$ could function as a tumor suppressor in cultured cells in vitro and in xenograft models in vivo [1-3]. Less explored has been whether TR $\alpha 1$ plays a role in carcinogenesis.

However, the article by Uchuya-Castillo et al in this issue reports the detection of increased expression of TR $\alpha 1$ in colorectal tumors of patient cohorts. Importantly, the THRA gene expression levels were significantly and directly correlated with Wnt activity. Previously, the same group reported that the ectopic expression of TR $\alpha 1$ in the intestine epithelium of $A p c^{+/ 1638 N}$ mice (vil$\mathrm{TR} \alpha 1 / A p c^{+/ 1638 N}$ mice) accelerated tumorigenesis with more aggressive tumor phenotypes [4]. Uchuya-Castillo et al have now further demonstrated that cellular TR $\alpha 1$ levels regulate Wnt activity to affect colon cancer cell proliferation and migration. The differential transcriptomic profiles in the adenocarcinomas of $A p c^{+/ 1638 N}$ mice and vilTR $\alpha 1 / A p c^{+/ 1638 N}$ mice further support the functional link of TR $\alpha 1$ to Wnt signaling. They found that increased expression of TR 1 was accompanied by decreased levels of several cellular inhibitors of Wnt signaling. Such inverse correlation found in the mouse models was also demonstrated in colorectal tumors of patient cohorts to account for how the elevated TR $\alpha 1$ led to the activation of Wnt signaling, thereby establishing the potential oncogenic role of TR $\alpha 1$ in the intestine epithelium.

The outcome of the present work highlights the contrasting functions of TR isoforms in tumorigenesis. Many lines of evidence have indicated that TR $\beta 1$ functions as a tumor suppressor. Loss of the THRB gene by chromosome deletion, as well as silencing in the expression of the THRB gene by promoter hypermethylation, has been reported in many human cancers. The tumor suppressing functions of TR $\beta 1$ have been demonstrated in many tumor cell lines derived from many cancers including thyroid cancer, breast cancer, and hepatocellular carcinoma. The compelling in vivo evidence to support the tumor suppressor role of TR $\beta 1$ came from a mouse model in which the loss of tumor suppressor functions by mutations causes spontaneous development of follicular thyroid cancer $\left(T h r b^{P V / P V}\right.$ mouse) [5]. However, it is important to point out that the mutated TR $\beta \mathrm{PV}$, identified in a patient with resistance to thyroid hormone (RTH $\beta$ ), does not act alone to initiate thyroid carcinogenesis. In the $T h r b^{P V / P V}$ mouse, TR $\beta P V$ collaborates and synergizes with other activated pathways such as thyroid stimulating hormone (TSH) [6] and PI3KAKT signaling [7] to induce the thyroid cancer phenotype [1]. In a similar fashion, TR $\alpha 1$ alone, as shown in vilTR $\alpha 1$ mice [4], does not initiate cancer development in the intestine epithelium. As shown in vil-TR $\alpha 1 / A p c^{+/ 1638 N}$ mice, synergy of elevated TR $\alpha 1$ with Wnt signaling leads to tumorigenesis. While TR isoforms exhibit contrasting roles in tumorigenesis, the common theme is that both isoforms, via diverse and extensive cross-talks with other cellular regulators, synergize with tumor promoters and/or attenuate tumor suppressor functions, leading to tumorigenesis.

Earlier, mice with single or double TR isoform knockout have clearly revealed that TR isoforms exhibit redundant functions as well as isoform-specific actions. That TR $\alpha 1$ and TR $\beta 1$ exhibit contrasting molecular actions in tumorigenesis has further expanded the scope of isoform-dependent actions in vivo, which would compel the need to address a long-standing important biological question as to why there are TR isoform-dependent actions in vivo. At present, little is known about the molecular basis underlying the isoform-dependent actions. In view of the findings that both TR isoforms necessitate the collaborations and synergy with other regulators critical in cellular functions to affect tumorigenesis, it would be reasonable to speculate that the divergent amino-terminal $\mathrm{A} / \mathrm{B}$ domains in the two isoforms could play an important role in dictating the isoform-dependent functions. The three-dimensional structure for each TR isoform, while currently unknown, could form distinct interacting surfaces to recruit different transcription factors and/ or enhancers on chromatin to affect gene transcription. The tissue-dependent expression of TR isoforms could further expand the combinatory networks, leading to the 
manifestation of TR isoform actions in a cellular-contextdependent manner. However, this critical question requires additional studies.

The findings reported by Uchuya-Castillo et al in this issue should prompt further studies to address several critical questions. 1). In patients with colorectal cancer as well as in vil-TR $\alpha 1 / A p c^{+/ 1638 N}$ mice, the increased TR $\alpha 1$ level is the key to activate Wnt signaling to induce tumorigenesis. Thus, it would be important to identify the cellular factors that could elevate TR $\alpha 1$ levels in intestine epithelium, ultimately leading to colorectal cancer. 2). Is there a defined threshold in the "elevated" TR $\alpha 1$ that could impact the development of colorectal cancer? 3). Can the elevated expression of TR $\alpha 1$ in other tissues in which a known oncogenic mutation is expressed promote cancer development? 4). Wnt is expressed in many tissues initially during development and later during growth. Can elevated TR $\alpha 1$ synergize with Wnt signaling in other tissues to impact tumorigenesis? These questions together with others yet to be defined could help to further our understanding the oncogenic actions of TR $\alpha 1$. The uncovering of the oncogenic actions of the elevated TR $\alpha 1$ in the intestine epithelium reported by Uchuya-Castillo et $a l$ is a beginning to spur additional studies.

Sheue-Yann Cheng: Laboratory of Molecular Biology, Center for Cancer Research, National Cancer Institute, Bethesda, Maryland, USA

Correspondence to: Sheve-Yann Cheng, email
chengs@mail.nih.gov

Keywords: thyroid hormone receptor isoforms; tumor suppressor; oncogene; Wnt signaling; TR $\beta$ mutations

Received: July 09, 2018

Published: August 10, 2018

\section{REFERENCES}

1. Zhu X, et al. Endocrinology. Springer, Cham. 2018; 233 257. https://doi.org/10.1007/978-3-319-44675-2_10.

2. Martínez-Iglesias O, et al. Cancer Res. 2009; 15:69:501-9. https://doi.org/10.1158/0008-5472.CAN-08-2198.

3. Martínez-Iglesias OA, et al. Proc Natl Acad Sci U S A. 2016; 113:E328-37. https://doi.org/10.1073/pnas.1520469113.

4. Kress E, et al. Gastroenterology. 2010; 138:1863-74. https://doi.org/10.1053/j.gastro.2010.01.041.

5. Suzuki H, et al. Thyroid. 2002; 12:963-9. https://doi.org/10.1089/105072502320908295.

6. Lu C, et al. Endocrinology. 2010; 151:1929-39. https://doi.org/10.1210/en.2009-1017.

7. Furuya F, et al. Proc Natl Acad Sci U S A. 2006; 103:17805. https://doi.org/10.1073/pnas.0510849103.

Copyright: Cheng et al. This is an open-access article distributed under the terms of the Creative Commons Attribution License 3.0 (CC BY 3.0), which permits unrestricted use, distribution, and reproduction in any medium, provided the original author and source are credited. 